\title{
"LA CIUDAD DE LOS COLORES": UNA EXPERIENCIA DE INTEGRACIÓN JUVENIL EN EL MARCO LOCAL DE UN BARRIO MARGINAL EN ALICANTE
}

\section{"THE CITY OF COLORS": AN EXPERIENCE OF YOUTH INTEGRATION IN THE LOCAL CONTEXT OF A MARGINAL NEIGHBORHOOD IN ALICANTE}

\author{
Virgilio Francisco Candela Sevila \\ Universidad de Alicante. España/Spain \\ virgilio.candela@gmail.com
}

Recibido/Received: 04/02/2015

Modificado/Modified: 21/04/2015

Aceptado/Accepted: 29/04/2015

\section{RESUMEN}

Este artículo explica el nacimiento, posterior evolución y gestión de un proyecto de intervención juvenil en la Zona Norte de la ciudad de Alicante nacido en 2007 y que permanece vigente dentro de un Plan Integral Municipal específico. Un proyecto gestionado por una entidad social local sin ánimo de lucro (Asociación Cultural Dánae) y que está formado por un conjunto de 80 personas entre alumnos, padres o tutores, monitores y coordinadores. El programa se desarrolla con el apoyo oficial del Ayuntamiento de Alicante y de la Generalitat Valenciana en un contexto vecinal degradado y con escasos recursos municipales (Zona Norte de la ciudad) que acoge un número muy importante de población inmigrante que, en algunos puntos, supera el $45 \%$ y donde conviven más de 120 nacionalidades. Es un proyecto muy reconocido socialmente y ha sido merecedor de premios municipales y regionales.

\section{PALABRAS CLAVE}

Juventud, interculturalidad, barrio marginal, convivencia, buena práctica, políticas públicas.

\section{SUMARIO}

1. Introducción. El marco general de la investigación. 2. La Ciudad de los Colores, programa integral de atención a la población infantil y juvenil de la Zona Norte de la ciudad de Alicante. 3. Conclusiones. Bibliografia.

\begin{abstract}
This article explains the birth, evolution and subsequent project management of a juvenile intervention in the Northern of the city of Alicante, born in 2007 and remains in effect within a specific Municipal Comprehensive Plan. A project managed by a local social organization nonprofit (Danae Cultural Association) and is formed by a group of 80 people, including students, parents or guardians, monitors and coordinators. The program is developed with the official support of the City Council of Alicante and the Generalitat Valenciana in a neighborhood context degraded and economically disadvantaged (North Zone of the city) which hosts a significant number of immigrants that in some neighborhoods, exceeds $45 \%$ and live more than 120 nationalities. It is a very socially recognized project and has been awarded by municipal and regional institutions.
\end{abstract}

\section{KEYWORDS}

Youth, multiculturalism, slum living, good practice, public policy. 


\section{CONTENTS}

1. Introduction. Framework of the research. 2. "The City of Colors", comprehensive care to children and youth in the Northern of the city of Alicante. 3. Conclusions. References.

\section{INTRODUCCIÓN. MARCO GENERAL DE LA INVESTIGACIÓN}

El proyecto analizado en este artículo tiene su razón de ser en un contexto ciudadano como el de la ciudad de Alicante, donde conviven actualmente hasta 144 nacionalidades distintas. Y más concretamente en un barrio o espacio vecinal, denominado Zona Norte, donde se concentran los porcentajes de población extranjera más altos de toda la ciudad. Este hecho motivó el diseño municipal de un Plan Integral de Recuperación de los Barrios de la Zona Norte (apoyado con un Proyecto URBAN en 2008) donde se incardina el programa "La Ciudad de los Colores". Trataremos a continuación de explicar brevemente el escenario principal de esta iniciativa social intercultural.

\subsection{La inmigración en la ciudad de Alicante}

Alicante cuenta en la actualidad con una población de 337.579 habitantes, un 14,7\% de los cuales son de origen extranjero (concretamente 49.721 personas, según el Padrón de Habitantes de 2014). Comparando los datos poblacionales de la ciudad lucentina respecto a 2000 observaremos un crecimiento de más de 57.000 personas. En ese año (2000) la población inmigrante era de 6.069 (un 2,2\%). Los hechos, pues, hablan por sí solos y demuestran que Alicante es una de las capitales de España con mayor crecimiento de población inmigrante a pesar de la crisis económica actual. Ésta, ha provocado regresos de ciudadanos de origen latinoamericano (argentinos, ecuatorianos, colombianos o uruguayos) pero en cambio el crecimiento superior de la llegada de población rumana y africana (Marruecos y Argelia).

En la actualidad, las nacionalidades extranjeras con mayor presencia en Alicante son, por este orden: argelina (6.288 personas), rumana (4.477), marroquí (3.916), italiana (3.281) y colombiana (3.144). Existe además una notable colonia europea (-la ya referida italiana-, francesa, alemana y británica) así como una importante presencia de personas del Este de Europa (Rusia, Bulgaria o Ucrania).

Estos datos difieren considerablemente respecto a la población inmigrante residente en la provincia de Alicante: el porcentaje de esta población alcanza el 24,1\% (469.417 extranjeros), siendo 297.808 comunitarios y 171.609 no comunitarios. Por países la presencia europea es notable, Reino Unido (130.541) y Alemania (35.916), seguidas de Marruecos (36.692), Rumanía (34.037), Países Bajos (17.192), Ecuador (15.150) o Colombia (13.826). Existe en la provincia de Alicante hasta 20 municipios con más de la mitad de su población de origen extranjero. Destacamos en este sentido las localidades de San Fulgencio (77,7\%), Rojales $(76,3 \%)$, Calpe (62\%), Alfás del Pí $(56,8 \%)$, Jávea $(54,4 \%)$, Torrevieja $(53,2 \%)$ y Orihuela (42,8\%).

Señalaría también que estamos inmersos en un evidente cambio de ciclo migratorio en la provincia de Alicante: en la actualidad son más los extranjeros que marchan que aquellos que llegan a nuestra tierra. Desde 2009 se ha ralentizado la llegada de población extranjera y se han estabilizado las cifras coincidiendo con la grave crisis económica que ha vivido España.

Volviendo a Alicante capital debemos destacar que la población inmigrante reside en mayor proporción en los barrios que conforman la Zona Norte de la ciudad. Así encontramos 
los porcentajes más altos en barrios como Colonia Requena (48,6\%), Virgen del Remedio (34,4\%), Sidi Ifni-Nou Alacant (29,5\%) o Juan XXIII (23,9\%). Este espacio vecinal, como su propio nombre indica, está situado en la parte norte de Alicante y tiene una personalidad propia que destacamos en el punto siguiente. El Programa "La Ciudad de los Colores" se desarrolla en Virgen del Remedio y tiene un ámbito de actuación global en toda la Zona Norte.

\subsection{La Zona Norte de Alicante}

Barrios de la Zona Norte es la denominación que recibe una zona de actuación municipal que comprende un total de seis barrios con una naturaleza socioeconómica muy similar y que se localiza en el límite norte de Alicante, a unos dos kilómetros del centro de la ciudad. Está conformado por los barrios de Virgen del Remedio, Colonia Requena, Virgen de Carmen, Juan XXIII, Cuatrocientas Viviendas y Sidi Ifni-Nou Alacant, y forma parte del área periférica de Alicante, cuestión que le dota de unas particularidades urbanísticas negativas.

Estos barrios nacieron en los años $50 \mathrm{y}$, principalmente, en la década de los 60, ante el crecimiento de la ciudad y la necesidad de vivienda por parte de los nuevos ciudadanos que procedían de la migración interna peninsular. Barrios surgidos en torno a las vías de comunicación existentes que carecían de los servicios municipales básicos. La ubicación desordenada de la periferia ha motivado una disposición radial del espacio, provocando la existencia de barrios inconexos que, unido a la edificación de alta densidad, ha provocado actualmente graves problemas urbanísticos y la carencia de equipamientos vecinales. En definitiva, un crecimiento irregular e imperfecto de un espacio municipal que no forma parte del Plan General de Ordenación Urbana de la ciudad.

Existen, por tanto, unos hechos evidentes, como el de marginalidad y exclusión social al referirse a la Zona Norte como consecuencia de la degradación urbanística, ambiental y social que comentamos. Esta situación de vulnerabilidad se manifiesta al conocer las principales carencias de la Zona Norte: un altísimo índice de desempleo (alcanza en estos barrios el $30 \%$ y el $40 \%$ de la tasa de paro), una tasa de actividad económica frágil, un alto nivel de pobreza y exclusión, un elevado número de inmigrantes y minorías étnicas, un bajo nivel educativo y un elevado índice de abandono escolar, un nivel alto de criminalidad y delincuencia y una degradación medioambiental de la zona, entre los aspectos más destacables.

La naturaleza decadente de estos barrios motivó la intervención municipal desde 2005, fecha en la que el Servicio de Coordinación de Áreas del Ayuntamiento de Alicante inició un minucioso estudio barrial que determinó resultados alarmantes en algunos espacios. Es el origen del Plan Integral de la Zona Norte, un ambicioso proyecto institucional donde se incardina el programa juvenil que estamos analizando.

Debemos señalar además que contrasta la marginalidad de este espacio ciudadano con la potencia de su tejido social y vecinal. Ya desde la llegada de la democracia el barrio de Virgen de Remedio se convirtió en el epicentro de las demandas vecinales ciudadanas y se libraron entonces duras pugnas por defender este espacio con el flamante Ayuntamiento. Hoy en día la Zona Norte congrega el mayor número de asociaciones juveniles, vecinales, culturales, religiosas e interculturales de toda la ciudad de Alicante y la voluntad y firme determinación de sus habitantes de luchar por estos barrios. 


\subsection{EI Plan Integral de la Zona Norte de Alicante}

El Plan Integral Barrios Vulnerables Zona Norte de Alicante tiene su origen a finales de 2004 con las demandas vecinales de los habitantes de la Zona Norte reclamando actuaciones que frenaran la situación de deterioro físico, económico y social que padecían y que estaban vinculados con la seguridad ciudadana, con cuestiones educativas y con la acción en espacios públicos. Desde entonces se desarrollan distintas reuniones entre representantes institucionales de la ciudad de Alicante, la Comunidad Valenciana y el Gobierno de España que desembocan en la firma en febrero de 2007 del "Acuerdo Marco entre la Conselleria de Territorio y Vivienda y el Ayuntamiento de Alicante para la intervención de rehabilitación urbana en los barrios de la Zona Norte de la ciudad de Alicante".

En marzo de 2007 (dos meses antes de la convocatoria electoral local) el gobierno municipal organizó un Taller de Trabajo, abierto a todo el tejido asociativo de la Zona Norte, donde se abrió un proceso participativo de propuestas y acciones para mejorar estos barrios y que se incluirían en el Plan Integral de la Zona Norte. Un año después, en 2008, el Plan Integral se verá enmarcado en la Iniciativa Europea URBAN de regeneración urbana de zonas degradadas, cofinanciada con fondos FEDER.

El presupuesto total del Plan Integral, contemplado para el periodo de 2007 a 2013, fue de 161 millones de euros, de los cuales 122 millones eran aportación directa de la Generalitat Valenciana, 18 millones del Ayuntamiento de Alicante, el Plan URBAN aportaba 17 millones, 2 millones de otras entidades y más de un millón procedente de los fondos FEDER.

Las estrategias y objetivos del Plan Integral han sido diseñadas en función de las carencias y posibilidades que ofrece el área de actuación. Destacamos las principales acciones:

a) Disminuir el abandono de la población de estos barrios mediante la rehabilitación de viviendas.

b) Mejorar la percepción social de la zona con un cambio de entorno mediante equipamientos y la integración cultural de la población inmigrante.

c) Reducir el absentismo escolar y elevar el grado de empleabilidad de la población en busca de empleo.

d) La inclusión social de colectivos con baja empleabilidad y hábitos culturales distintos del entorno.

e) Apoyar el comercio y la economía de la zona y crear un Mercado Municipal en el centro de influencia que será un dinamizador económico y social del entorno.

f) Potenciar el nuevo trazado del tranvía para descongestionar el tráfico rodado.

g) Coordinar y aproximar los equipamientos de los cuerpos de policía para mejorar la seguridad de la zona.

Así mismo, las estrategias del proyecto se centran en cinco puntos fundamentales:

a) Hacer un barrio físicamente atractivo e integrado con el resto de la ciudad

b) Formación para el empleo y fomento del espíritu empresarial

c) Seguridad Ciudadana

d) Inserción socioeducativa, familiar y salud comunitaria

e) Integración cultural, convivencia y participación ciudadana

El Programa "La Ciudad de los Colores" se incluyó en el texto final del Plan Integral de la Zona Norte a través de su vertiente musical, conocida públicamente como la Escuela de Música Intercultural de Alicante. El proyecto fue avalado por las Concejalías de Acción Social y de Presidencia y Coordinación de Áreas, y fue dotado con un presupuesto para el periodo $2008-2013$ de $150.000 €$. 


\section{2. "LA CIUDAD DE LOS COLORES", PROGRAMA INTEGRAL DE ATENCIÓN A LA POBLACIÓN INFANTIL Y JUVENIL DE LA ZONA NORTE DE LA CIUDAD DE ALICANTE}

\subsection{La identificación y la descripción del programa. El ámbito de actuación y descripción}

El Programa Intercultural de Intervención Juvenil de la Zona Norte "La Ciudad de los Colores" pretende dar una cobertura socioeducativa a un sector vulnerable de la sociedad alicantina, como es la juventud de los barrios de la Zona Norte. Esta labor que se realiza desde la Asociación Cultural Dánae complementa la educación formal que se recibe en los centros educativos municipales y cubre posibles carencias educativas en el plano no formal, es decir, que provengan de aquella parte de la formación que una persona va adquiriendo desde niño en su casa, en la calle, en el contacto con la sociedad, y que conforma su condición de ciudadano.

Desde Dánae se entendió que dicha labor socioeducativa debía realizarse de forma integral, abarcando todos aquellos ámbitos de la vida que toda persona debe conocer en su proceso de educación. Ahora bien, dada la naturaleza de este proyecto y su inherente vinculación a la Zona Norte de la Alicante, el plan de actividades que contiene es especialmente sensible a aquellos problemas que reviste esta parte de la ciudad, y que han quedado bien identificados en documentos como el Plan Integral de la Zona Norte y el Plan URBAN para los Barrios de la Zona Norte.

Si bien desde la entidad son conscientes de las debilidades de estos barrios, también se han tenido muy en cuenta aquellas características que los convierten en un espacio vecinal valioso y de futuro. Una de estas características positivas es la diversidad de nacionalidades y razas que habitan la Zona Norte, lo cual es uno de los motivos de este Programa Intercultural de Intervención Juvenil.

Los contenidos fundamentales de este programa vienen desarrollándose satisfactoriamente desde hace años, es por eso que no ha variado mucho su estructura respecto a 2007. Las charlas, talleres, excursiones y dinámicas realizadas con los jóvenes que integran el proyecto y con sus padres y tutores les ha permitido optimizar día a día este programa haciéndolo cada vez más interesante para ellos y más completo para su formación.

Este proyecto atiende una programación de actividades diversas que pueden ser agrupadas en ocho bloques temáticos, como son:

a. Solidaridad, Tolerancia e Interculturalidad.

b. Participación Ciudadana.

c. Acción Social y Prevención.

d. Medio Ambiente.

e. Comunicación y Nuevas Tecnologías.

f. Historia, Arte y Cultura.

g. Deporte y Vida Saludable.

h. Otras Actividades, Salidas y Excursiones.

Cada uno de estos bloques de materias señaladas incluye a su vez varios epígrafes, en los cuales se clasifican cada una de las concretas actividades que se desarrollan en el programa.

\subsection{Fundamentación del programa, duración y organización}

El Programa "La Ciudad de los Colores" nació hace casi siete años como programación formativa complementaria a la Escuela de Música Intercultural de Alicante, proyecto de la 
Asociación Cultural Dánae que forma parte del Plan Integral de la Zona Norte. Dicho programa ha ido creciendo a lo largo de este tiempo con el aumento del número de actividades realizadas y de los participantes de las mismas.

Entendemos que la génesis de esta iniciativa tiene mucho interés y por ello se le dedican algunas líneas. En julio de 2007 nació oficialmente el proyecto de la Escuela de Música Intercultural de Alicante desde la Asociación Cultural Dánae y con el apoyo explícito de las Concejalías de Acción Social y de Participación Ciudadana del Ayuntamiento de Alicante. Aquel proyecto, cuyo objetivo principal se explica más adelante, durmió el "sueño de los justos" desde septiembre de 2006 en los cajones de los técnicos municipales de las Concejalías de Acción Social y Juventud. La razón: la convocatoria de elecciones municipales en mayo de 2007 y lo incierto del resultado final. Pasadas las elecciones y reelegidos en sus cargos los antiguos concejales, el proyecto se retomó y se puso en marcha el sábado 7 de julio con una fiesta y un pasacalle en la Plaza de Argel del Barrio de Virgen de Remedio.

Aquella velada donde hubo mucha música (percusión brasileña), capoeira, baile, samba y pagoda, fue un espaldarazo respecto al futuro Plan Integral de la Zona Norte, todavía en proceso de redacción, y que carecía hasta de logos identificativos de su naturaleza. La Zona Norte se vio entonces sorprendida por una iniciativa de esencia lúdica y festiva que ponía su acento en los jóvenes y que pretendía la educación en valores de sus participantes a través del aliciente de una práctica musical inédita en ese espacio vecinal.

Tuvo la Escuela de Música un reconocimiento nacional al recibir la visita en agosto de ese mismo año (un mes después de su nacimiento) de Marquinhos Brown, hermano del célebre percusionista Carlinhos Brown, y que vino acompañado de otros músicos del autor carioca para conocer y apoyar expresamente la iniciativa alicantina recién presentada. Fue una verdadera locura la llegada de los jóvenes brasileños en Alicante por la presencia de medios televisivos y periodísticos de todo el país. Marquinhos Brown visitó la Escuela, enseñó a los chavales percusión, realizó un improvisado concierto y dio infinidad de entrevistas para los medios presentes. Su viaje a Alicante tenía el objetivo de impulsar la actividad local con el visto bueno de Carlinhos Brown.

Se estudió desde entonces la posibilidad de un hermanamiento entre las escuelas de música de Alicante y de Candeal (Salvador de Bahia). Para ello incluso la Concejalía de Acción Social no escatimó esfuerzos y concedió una subvención a la sede local de $18.000 €$. Meses después el propio Carlinhos Brown se encerró en su estudio a componer canciones y declinó la posibilidad de apadrinar la Escuela de Música de Alicante. El proyecto sufrió entonces un duro golpe moral que motivó el paso de la euforia municipal inicial a una situación de respeto y apoyo al proyecto pero sin el interés despertado a sus comienzos.

La Escuela de Música continuó su labor sin descanso y hasta tuvo posibilidad de recibir la visita de los conocidos percusionistas de STOMP. En octubre de 2008, el proyecto fue conocido por los responsables de la Escuela de Música de Pracatum y por los Niños de Candeal de Brasil en su paso por Alicante dentro de la iniciativa de Solidaria, un programa organizado por la Conselleria de Inmigración y Ciudadanía de la Generalitat Valenciana. El alcance inicial de la Escuela de Música Intercultural de Alicante fue tal que logró en diciembre de 2008 el Premio Francisco Liberal del Ayuntamiento de Alicante en su vertiente de Participación Ciudadana.

Desde entonces, la relación entre las Escuelas de Alicante y Salvador de Bahia fue muy estrecha, aunque no se llegó a formalizar oficialmente por la provisionalidad del centro brasileño que fue cerrado en 2010 por un nuevo espacio de formación musical municipal. Ello no impidió que profesores de Pracatum visitaran regularmente Alicante para dar clases 
de música o de creación artística a los jóvenes alicantinos (es el caso de los profesores Chrispin Junior o Ives Quaglia).

Como antes escribía, "La Ciudad de los Colores" nació a mediados de 2008 como programación complementaria a la Escuela de Música Intercultural de Alicante. Surgió como proyecto inicial de educación en valores de los jóvenes participantes en la Escuela de Música. Fue en realidad una nueva estrategia de la dirección del proyecto al advertir las dificultades que empezaban a tener al ser equiparado su proyecto inicial como una iniciativa de exclusiva naturaleza artística y musical y no como un programa integral de formación juvenil. Ello motivó la división del proyecto original en dos para concurrir a las subvenciones públicas municipales que además se tornaron incompatibles desde diferentes concejalías.

La Escuela de Música era en realidad un proyecto de integración juvenil de los niños de este enclave tan importante de la ciudad de Alicante. Con el "pretexto" de la música, el baile, el teatro, la animación y la percusión, se pretendía fomentar valores de ocio sano y saludable entre los chavales de la Zona Norte, aumentar su autoestima y hacerles sentir importantes. El fin último de esta iniciativa no es otro que la formación integral de los chicos que formaban parte del proyecto y su participación en las iniciativas ciudadanas de la Zona Norte. El proyecto original pretendía armonizar una labor de recuperación e integración de la población juvenil de la Zona Norte a través de la expresión artística, sin descuidar el objetivo final de esta interacción: la solidaridad, la tolerancia, el respeto por otras culturas y el compromiso por formar parte de un movimiento juvenil, que les permitiera integrarse positivamente en su entorno socio-cultural. La intención final de este proyecto era la formación integral de los jóvenes vecinos de la Zona Norte y su total integración en las instancias vecinales y sociales surgidas de él.

Desde su nacimiento, el Programa "La Ciudad de los Colores" se erigió en el proyecto más amplio y ambicioso de la Asociación Cultural Dánae en la Zona Norte de la ciudad de Alicante e integró a la Escuela de Música en su seno.

Como ya se ha comentado, el proyecto surgió de la necesidad de ofrecer a los jóvenes de la Zona Norte una serie de herramientas sociales que les permitiera afrontar el futuro de su crecimiento con seguridad, madurez y bienestar. En los más de cuatro años de trabajo diario en los barrios de la Zona Norte, los voluntarios de la Asociación Cultural Dánae han podido percibir los "alicientes" que para los jóvenes de esta edad tiene la calle: libertad, independencia familiar, ausencia de normas y límites horarios, posibilidad de consumo libre de tabaco y bebidas alcohólicas, impunidad a la hora de asaltar personas, robos, violencia, etc. Son diarios los ejemplos que en este sentido observan los jóvenes de la Zona Norte en los alrededores del Centro Social Gastón Castelló y hasta en alguna ocasión dentro del propio Centro.

El objetivo original de este programa radicaba en la necesidad de aportar una serie de valores sanos y solidarios a unos jóvenes que se adentraban en la adolescencia careciendo de las herramientas necesarias para entender qué les estaba ocurriendo. Valores sencillos, elementales, pero fundamentales: respeto (a sus compañeros y a los profesores), educación, disciplina, trabajo en equipo, deseo de aprender, humildad, constancia, superación. Valores que en muchos casos desconocían por no practicarlos en casa o bien por "relajación" familiar pero que son muy necesarios para la completa formación de los jóvenes. En ese sentido ha girado siempre la actividad de Dánae, en la creación de personitas libres y responsables capaces de entender el mundo en el que viven con todas las injusticias sociales que ello conlleva. 
En palabras de uno de sus responsables "hemos ido regando una semillita de amor y de solidaridad en sus cabecitas y corazones desde hace años para que fueran buenas personas, alegres, sencillas, comprometidas con su barrio y con sus vecinos, respetuosas con las gentes que viven en él, con el medio que les rodea, personas inquietas con opinión sobre lo que ocurre en el mundo, personas que fomenten la interculturalidad y que defiendan las causas nobles y justas de las personas que más lo necesitan. Todo esto, en definitiva, resume el objetivo principal de "La Ciudad de los Colores" y a ello dedica la entidad altruistamente dos días a la semana todos los meses del año".

La justificación: la descripción de los factores que dan origen al mismo.

a) El alto índice de población inmigrante. Esta es sin duda una de las circunstancias positivas que motivan la intervención de Dánae en la Zona Norte con este proyecto. A su juicio, la heterogeneidad en la población es un factor positivo, pues constituye una mayor riqueza cultural. Sin embargo, este factor genera en muchos casos una visión negativa de esta zona de la ciudad. Así pues, Dánae pretende ahondar en la promoción de valores de convivencia y tolerancia entre la población más joven de la Zona Norte y difundir una visión más positiva de este sector de la ciudad, lo cual haría justicia a una situación de interculturalidad.

La entidad organiza además, dentro de su programa de trabajo anual, proyectos de evidente cariz intercultural donde se promueve el respeto a todas las personas y culturas internacionales que residen hoy en día en Alicante. Los jóvenes de "La Ciudad de los Colores" han participado siempre de forma activa en estos proyectos (hablamos de las Marchas Cívicas Participativas, del Poema Gigante Intercultural ó del Programa de Voluntariado Medioambiental Intercultural en Guardamar del Segura.

b) El bajo nivel educativo y el absentismo escolar. Muchos niños y jóvenes de la Zona Norte tienen padres con largas jornadas laborales que les impiden tener un mayor control sobre sus hijos. En otros casos, las familias se encuentran desestructuradas, o a los padres se les ha privado de la patria potestad de sus hijos. Este distanciamiento permite a muchos niños y jóvenes de la Zona Norte vivir sin las rutinas propias de cualquier persona de su edad, lo que en muchos casos se traduce en absentismo escolar o muy bajo rendimiento académico. La programación de este proyecto trata de aportar rutinas fijas que disciplinen el crecimiento y la educación de los participantes del programa.

c) Riesgo de exclusión social elevado. La multitud étnica o el bajo nivel económico de muchas familias de la Zona Norte son factores que crean una potencial exclusión social o, en otros casos, una exclusión social efectiva sobre determinados colectivos.

El conjunto de actividades programadas, especialmente aquellas que ponen en contacto a los jóvenes del programa con entidades y agentes sociales o las que implican el conocimiento de nuevos lugares de la ciudad, son una forma unívoca de paliar estas situaciones.

d) Elevado índice de inseguridad ciudadana. La prevención de conductas nocivas y/o ilícitas es una preocupación de la Asociación Cultural Dánae que se ve reflejada implícita o explícitamente en muchas de las actividades programadas. En la Zona Norte existe, cierto es, un índice importante de inseguridad ciudadana, pero este es un factor que no debe ser estereotipado ni magnificado en modo alguno y que, además, es prevenible con la educación necesaria.

\section{La duración.}

El proyecto "La Ciudad de los Colores" nació en 2008 y continúa aún su desarrollo en los momentos de redactar este artículo. Su programa de actividades se diseña semestralmente pero tiene un alcance anual. Las clases tienen lugar los martes de 18 a 20 horas y los sábados 
de 10 a 14 horas en el Centro Social Comunitario Gastón Castelló de la Concejalía de Acción Social del Ayuntamiento de Alicante. El proyecto descansa en los mismos periodos vacacionales del calendario escolar (Semana Santa, Verano y Navidades).

Las instalaciones del centro municipal son excelentes, con un salón de actos muy amplio que posee escenario teatral (idóneo para el ensayo musical), con un gimnasio pequeño pero bien arreglado (para clases de baile y capoeira) y con un espacio juvenil compuesto de dos aulas que son utilizadas para la realización de talleres diversos.

\subsection{La financiación del programa}

La previsión inicial de la Escuela de Música de formar parte del Plan Integral de la Zona Norte se quedó en anécdota y más la dotación económica prevista para el periodo 2007-2013 de 150.000 euros. Ello motivó desde un primer momento la necesidad de concurrir a las subvenciones públicas del Ayuntamiento de Alicante, de la Generalitat Valenciana y de la Universidad de Alicante. El presupuesto total que ha obtenido el programa es de $110.000 €$, en proporción de unos 13.750 euros anuales. Una financiación importante pero a todas luces insuficiente para poder gestionar correctamente un programa que abarca a más de 70 personas al año en un calendario de enero a diciembre y con múltiples actividades mensuales.

La negativa municipal a que el proyecto formara parte de la financiación del Plan URBAN motivó el freno de las aspiraciones iniciales del proyecto que pretendía convertir el Centro Social donde se desarrollaba en un gran espacio de convivencia e interacción juvenil.

Se estima pues que el presupuesto total obtenido por este proyecto por parte de la Asociación Cultural Dánae alcanza los $110.000 €$ en el periodo comprendido entre 2007 y 2014. En un 71\%, la principal institución financiadora ha sido el Ayuntamiento de Alicante en sus Concejalías de Acción Social y Coordinación de Proyectos, principalmente, y en menor medida, de Participación Ciudadana. En un $8 \%$ de ese importe podemos estimar la colaboración por parte de la Universidad de Alicante (desde su Vicerrectorado de Alumnado y desde el Consejo de Alumnos de la Universidad) así como la aportación de la Generalitat Valenciana (en su Conselleria de Bienestar Social). Podemos cifrar la aportación de la Obra Social de la extinta Caja de Ahorros del Mediterráneo en un 3\% aproximadamente. Por último, la propia entidad ha aportado un $10 \%$ de recursos propios en su proyecto intercultural.

Una de las principales fortalezas del proyecto es que cuenta con una nómina importante de voluntarios que ejercen la labor de coordinación de las actividades del programa. Ese voluntariado intercultural ha permitido que el proyecto sobreviva año tras año al invertir todos los fondos obtenidos en recursos para los propios jóvenes.

Los ingresos obtenidos se invierten anualmente en una serie de capítulos que no divergen mucho de año a año. Podríamos señalar que de un 100\% total se destina a Personal un $10 \%$, a Gastos Administrativos un 15\%, a Gastos de Transporte (autobuses y furgonetas) un 25\%, a Gastos en Materiales Didácticos un 20\%, a Gastos en Indumentaria un 15\%, a Gastos de Instrumentos Musicales y repuestos un 20\% y un 5\% a Alimentación (almuerzos semanales los sábados, tras las actuaciones públicas, etc.).

Es sumamente significativo cómo ninguna obra social financiera local haya decidido subvencionar este programa a pesar de su vistosidad y de tener en los jóvenes el centro de sus operaciones. En un principio fue así por el deseo de la Caja de Ahorros del Mediterráneo de financiar las iniciativas incluidas primero en el Plan Integral de la Zona Norte de Alicante y, después, en el Plan URBAN. Posteriormente se denegó sistemáticamente la colaboración directa de "La Ciudad de los Colores" desde la Convocatoria de CAM Integra (donde se 
contaba con un presupuesto total de 3'5 millones de euros) al destinarse la mayor parte de los fondos a proyectos para asociaciones de trabajo con personas discapacitadas o bien a proyectos cuyos promotores participaran de las redes clientelares de la Obra Social.

\subsection{Los objetivos y los recursos disponibles}

Los objetivos generales de proyecto son:

a) La formación socio-educativa y educación en valores de un sector de la población alicantina tan vulnerable como la juventud de la Zona Norte.

b) La acción social en su vertiente preventiva de todos aquellos factores que afectan a los niños y jóvenes de la Zona Norte tal y como se describe en el Plan Integral de la Zona Norte y en el Plan URBAN.

c) La cobertura a las necesidades de los sectores prioritarios de los Servicios Sociales de la ciudad de Alicante (familia, infancia, juventud e inmigración).

Los recursos de los que se dispone:

La Asociación Cultural Dánae forma parte del Consejo de Alumnos de la Universidad de Alicante. En sus dependencias dispone de salas de reuniones, ordenadores, biblioteca así como de asesoramiento en materia juvenil por parte de los técnicos universitarios. Igualmente, Dánae está inscrita en el Registro de Entidades de Interés Municipal (R.E.I.M.) del Excmo. Ayuntamiento de Alicante y dispone de todos los servicios que presta la Concejalía de Participación Ciudadana.

La entidad participa en la mesa de trabajo del S.A.I. de la Concejalía de Acción Social, pertenece al Consell de la Joventut d'Alacant y está inscrita en la AECI, disfrutando de los recursos técnicos que estas entidades presta regularmente a las asociaciones inscritas.

Desde 2010, Dánae participa activamente en la mesa de trabajo de Virgen de Remedio creada por el Equipo de Intervención Comunitaria de la Zona Norte. En este grupo se han analizado los principales problemas que presenta el barrio y se han empezado a diseñar las posibles intervenciones a desarrollar en la zona. El grupo de jóvenes integrantes del Programa "La Ciudad de los Colores" logró, por dos ocasiones sucesivas, el primer premio en el Concurso de Vídeos Jóvenes organizado por la mesa en sus primeras ediciones.

Los programas de la entidad en la Zona Norte se remontan a 2007. Desde entonces el número de usuarios inscritos en sus servicios y programas (305 jóvenes y 100 padres en el total de la intervención, alrededor de 35 jóvenes y de 15 padres y tutores actualmente) garantiza el grado de implantación de la entidad en este espacio vecinal y la excelente relación con la Concejalía de Acción Social. Mencionar por último que la coherencia técnica y de gestión de la Asociación Cultural Dánae fue destacada con la obtención del Premio a la Solidaridad y al Voluntariado 2008 de la Generalitat Valenciana.

El Programa "La Ciudad de los Colores" cuenta con un equipo de trabajo integrado por siete personas de diversa cualificación y con unas responsabilidades muy determinadas. Se detallan a continuación:

a) 1 responsable general del proyecto. Gestor cultural, historiador y técnico en la gestión de políticas públicas migratorias e interculturales.

b) 1 coordinador general del proyecto. Licenciado en derecho y experto en materia intercultural jurídica.

c) 1 administración general del proyecto.

d) 1 animador sociocultural titulado.

e) 3 voluntarios de la Asociación Cultural Dánae. Su función es la planificación del proyecto y las gestiones necesarias para llevarlo a cabo. Voluntarios de muy diversa 
formación: trabajadores sociales, estudiantes de magisterio infantil, y animadores socioculturales.

\subsection{La evaluación del programa}

Desde la Asociación Cultural Dánae, con el fin de poder atender todas las actividades del programa con la máxima calidad humana, se fijó un límite de inscripciones en el programa "La Ciudad de los Colores". Así pues, se admiten como máximo a 40 niños y jóvenes, que serán los beneficiarios directos del programa.

La inscripción está abierta a niños y jóvenes de entre 10 y 18 años -ambos inclusive- de cualquier punto de la ciudad de Alicante aunque, basándose en la experiencia de los años de andadura del programa, las inscripciones son de jóvenes pertenecientes a alguno de los barrios de la Zona Norte.

No hay más requisito de inscripción que la escolarización y el compromiso individual y familiar de acatamiento de las normas del programa, fundamentadas en el respeto y el civismo. Los participantes son inscritos en riguroso orden de petición. La inscripción, así como la participación en la totalidad de las actividades programadas, no tienen coste económico alguno para los beneficiarios.

Los factores de riesgo que puedan influir en el desarrollo del proyecto vienen determinados por las mismas circunstancias del colectivo de atención que se pretenden mejorar. Estos factores pueden ser:

a) La falta de una rutina y disciplina en los hábitos de vida. Esto dificulta la asistencia regular de algunos jóvenes a todas las actividades programadas.

b) La falta de apoyo de los padres o tutores de los niños y jóvenes que participen en el programa.

Para el primer caso se ha previsto un sistema de advertencias sobre las faltas de asistencia reiteradas e injustificadas que, llegado a un límite fijado y acordado por el equipo de responsables del programa, podrá acarrear la expulsión del mismo.

Para abordar la segunda problemática el equipo de responsables de Dánae implicados en el programa se planteó en 2009 la creación de una "Escuela de Padres". Esta iniciativa pretende la ejecución de una serie de reuniones regulares con todos los padres o tutores de los niños y jóvenes que participan en el programa para informar de forma cercana sobre la marcha de las actividades, y crear una conciencia en los padres sobre el programa "La Ciudad de los Colores" que conduzca a una implicación activa o, cuanto menos, a una valoración del programa.

La experiencia obtenida con los padres han sido muy satisfactorios y ha motivado a la entidad a seguir adelante con esta ingente labor al sentirlos plenamente integrados en la educación de sus hijos. Además, estas reuniones sirven para detectar posibles carencias educativo-afectivas que puedan existir en el seno de algunas familias, lo cual mejora el trato en los niños o jóvenes afectados en el programa. En este sentido debemos comentar que han sido derivados algunos casos de jóvenes participantes del proyecto a la psicóloga y trabajadora social del Centro Social Gastón Castelló

\section{CONCLUSIONES}

Con este trabajo se ha pretendido conocer la génesis y organización de un proyecto de intervención juvenil en un barrio degradado gestionado por una entidad local sin ánimo de 
lucro. Una iniciativa que surgió en un espacio vecinal de alto índice de población inmigrante antes de la aplicación de un Plan Integral de acción municipal. El proyecto, con unas potencialidades enormes al ser pionero en el barrio, trabajar con jóvenes y tener su temática principal en la interculturalidad, contó con el apoyo institucional del Ayuntamiento de Alicante desde un primer momento porque además abría unas posibilidades de expansión internacional al poder ser apadrinado por una figura de la música brasileña (Carlinhos Brown). La negativa del artista a apoyar la Escuela de Música provocó el desánimo político local motivando no el abandono del proyecto pero sí en cambio su no inclusión en la financiación directa de Plan Integral y frenando su crecimiento natural.

A día de hoy "La Ciudad de los Colores" es una iniciativa reconocida en la ciudad de Alicante y muy respetada por los vecinos de la Zona Norte. Es posiblemente el único proyecto de los referidos a jóvenes en la Zona Norte que mejores resultados está teniendo por la naturaleza educativa del mismo y por contar con un número importante de jóvenes que han permanecido en el proyecto desde su creación en 2007. Estos jóvenes se ven atraídos por lo que más les gusta (tocar percusión) pero asumen de buen grado la vertiente formativa del proyecto y participan activamente en las actividades organizadas por la dirección.

Para los voluntarios de Dánae el trabajo anual con estos chavales es agotador pero realmente enriquecedor. Horas y horas de trabajo en pro de la libertad personal, de la interculturalidad y la convivencia, de la participación ciudadana, del diálogo y la tolerancia se recogen ahora en forma de halagos por parte de los educadores y técnicos que conocen a los jóvenes y se sorprenden de su iniciativa, participación y buen comportamiento. Viniendo de un barrio con tantas dificultades sociales y económicas es digno de reseñar.

El proyecto está camino de cumplir ocho años de vida. Su futuro, pese a la solidez de su estructura organizativa, está en el aire por la dureza de la crisis económica y por la falta de apoyo específico del Ayuntamiento de Alicante.

Esta experiencia y caso práctico estudiado, pone en mi opinión de manifiesto dos hechos evidentes y relevantes en materia de gestión pública local:

a) La externalización de algunos servicios públicos locales y la colaboración en este caso no de la iniciativa privada con ánimo de lucro, sino de una asociación del llamado "tercer sector". Por tanto, esta colaboración es posible y necesaria en ciertos ámbitos municipales como el sociocultural.

b) El papel del voluntariado como colaboración desinteresada en la gestión pública en general, y en especial en el ámbito local

\section{BIBLIOGRAFÍA}

ALICANTE. Padrón de Habitantes 2000, 2010 y 2014. Ayuntamiento de Alicante.

ARIÑO VILLARROYA, A. (dir.) (2001). La ciudadanía solidaría. El voluntariado y las organizaciones de voluntariado en la Comunidad Valenciana. Valencia: Bancaja.

ASOCIACIÓ JUVENIL Alicantina de Estudios Sociales (1999). Investigación participativa sobre el asociacionismo y juventud en Alicante. Alicante: Ayuntamiento de Alicante, Concejalía de Juventud.

DEL ALCÀZAR GARRIDO, J. y CANDELA SEVILA, V. (2003). "La immigració llatinoamericana al País Valencià" en Revista Saitabi. Valencia: Universitat de València, Volumen LIII, pp. 149-172.

ESTRADA, Ó. (1993). El asociacionismo en Alicante. Alicante: Ayuntamiento de Alicante, Concejalía de Cultura.

GÓMEZ GIL, C. (2005). "Evolución de la población extranjera en la Comunidad Valenciana. Período 1998-2005" en Observatorio de la Inmigración de Alicante. Alicante: Universidad de Alicante. 
GÓMEZ GIL, C. (2006). "Multiculturalidad, cambios sociales e inmigración en la Comunidad Valenciana", en A. Piqueras (coord.) Mediterrània Migrant. Les migracions al País Valencià. Castellón: Universitat Jaume I, pp. 129-152.

INFORME 2011 (2011). Aproximación estadística a la inmigración en Alicante. ASTI-Alicante, 29 de julio. Secretariado Diocesano de Migración de Orihuela-Alicante.

INFORME 2014 (2014). Aproximación estadística a la inmigración en Alicante. ASTI-Alicante, 30 de junio. Secretariado Diocesano de Migración de Orihuela-Alicante.

PLAN (2008). Integral de Recuperación Barrios Zona Norte. Alicante.

PROYECTO URBAN (2009). Barrios Zona Norte de Alicante. Iniciativa URBAN 2007-2013 (2009). VALERO, J. R. (1992). La inmigración extranjera en Alicante. Alicante: Diputación de Alicante.

\section{Breve currículo:}

\section{Virgilio Francisco Candela Sevila}

Licenciado en Historia por la Universidad de Alicante (1996). Realizados los cursos de doctorado en Historia Contemporánea. Lectura de la Memoria de Licenciatura titulada "La transición a la democracia en la provincia de Alicante: de la oposición moderada a las primeras elecciones generales" (2003, Sobresaliente por unanimidad). Publicado el libro: Del franquismo a la democracia. La formación de la UCD en la provincia de Alicante (2007). Premio Extraordinario de Licenciatura (2005). Título de Inglés de la Escuela Oficial de Idiomas de Alicante. Grau Mitjà de Coneiximents en Valencià, Junta Qualificadora. Técnico y documentalista en la Biblioteca de Historia de la Biblioteca Virtual "Miguel de Cervantes" de la UA. Máster en la Gestión de Políticas Públicas Migratorias, Interculturalidad, Mediación y Cooperación. Máster en Liderazgo Político y Dirección Pública de Instituciones PolíticoAdministrativas. Gestor cultural en el Consejo de Alumnos de la UA (2004-2007). 